\title{
The COPD assessment test correlates well with the computed tomography measurements in COPD patients in China
}

This article was published in the following Dove Press journal:

International Journal of COPD

5 March 2015

Number of times this article has been viewed

\section{Yan Zhang* \\ You-Hui Tu* \\ Guang-He Fei}

Pulmonary Department, First Affiliated Hospital of Anhui Medical University, Hefei, Anhui, People's Republic of China

*These authors contributed equally to this work
Correspondence: Guang-He Fei

Pulmonary Department, First Affiliated Hospital of Anhui Medical University, Hefei 230022, Anhui, People's Republic of China

Tel +8655162922013

Fax +8655I 63635578

Email guanghefei@I26.com
Background: The chronic obstructive pulmonary disease (COPD) assessment test (CAT) is a validated simple instrument to assess health status, and it correlates well with the severity of airway obstruction in COPD patients. However, little is known about the relationships between CAT scores and quantitative computed tomography (CT) measurements of emphysema and airway wall thickness in COPD patients in the People's Republic of China.

Methods: One hundred and twelve participants including 63 COPD patients and 49 normal control subjects were recruited. All participants were examined with high-resolution CT to get the measurements of emphysema (percentage of pixels below $-950 \mathrm{HU}$ [\%LAA-950]) and airway wall thickness (wall area percentage and the ratio of airway wall thickness to total diameter). Meanwhile, they completed the CAT and modified Medical Research Council questionnaire independently.

Results: Significantly higher CAT scores and CT measurements were found in COPD patients compared with normal control subjects $(P<0.05)$, and there was a tendency of higher CAT scores and CT measurements with increasing disease severity measured by GOLD staging system. Positive correlations were found between CAT scores and CT measurements $(P<0.01)$. Using multiple linear stepwise regression, CAT score $=-46.38+0.778 \times($ wall area percentage $)+0.203 \times$ (\%LAA-950) $(P<0.001)$. Meanwhile, CAT scores and CT measurements in COPD patients all positively correlated with the modified Medical Research Council grades and negatively correlated with $\mathrm{FEV}_{1} \%(P<0.01)$.

Conclusion: CAT scores correlate well with the quantitative CT measurements in COPD patients, which may provide an imaging evidence that the structural changes of the lungs in this disease are associated with the health status measured by CAT.

Keywords: chronic obstructive pulmonary disease, pulmonary function, quantitative computed tomography

\section{Introduction}

The chronic obstructive pulmonary disease (COPD) assessment test (CAT) is a simple, validated, patient-completed questionnaire to assess and quantify health status and symptoms burden in COPD patients. ${ }^{1,2}$ It comprises eight questions, each presented as a semantic six-point (0-5) differential scale, providing a total score out of 40 . Scores of 0-10, 11-20, 21-30, and 31-40 represent mild, moderate, severe, or very severe clinical impact, respectively. ${ }^{3}$ The CAT has good internal consistency and test-retest reliability; ${ }^{1}$ previous studies have shown its significance in diagnosis, response to therapies, and management both in stable and exacerbating COPD patients. ${ }^{4-8} \mathrm{CAT}$ scores correlate well with important parameters of the disease, such as dyspnea grades, pulmonary function parameters, etc. ${ }^{7-9}$ Nowadays, it is widely used in clinical practice all over the world. 
COPD is a chronic lung disease, and it can lead to certain irreversible structural changes in the lungs, including airway wall remodeling and emphysematous lung destruction. ${ }^{10}$ With the advances both in computed tomography (CT) technology and computer software, the multi-slice spiral CT has not only improved the early diagnosis of pulmonary microstructures and morphologic changes, but also made the quantitative measurements of structural changes in the lungs possible. ${ }^{11-13}$ Since the high-resolution CT (HRCT) technology has a good spatial resolution, it is currently a better imaging technology to observe small airway and lung tissue disease. ${ }^{14}$ Studies have demonstrated that -950 Hounsfield units (HU) in the inspiratory phase is a suitable threshold for the measurements of retention volume in emphysema, ${ }^{15,16}$ and the ratio of airway wall thickness to total diameter (WT/D) and wall area percentage (\%WA) from CT scans can represent the airway dimensions in COPD patients. ${ }^{17,18}$ Moreover, researchers have conducted a series of studies on the relationships between disease-specific parameters and quantitative CT measurements. ${ }^{18-20}$ As far as we know, little is known about the relationships between CAT scores and quantitative CT measurements of emphysema and airway wall thickness in COPD patients in the People's Republic of China.

In the present study, we hypothesized that CAT scores might be related to the extent of emphysema and airway wall thickness in COPD patients. By measuring the extent of emphysema and airway wall thickness with HRCT, and investigating the relationships between CAT scores and quantitative CT measurements, we explored if there is any association of CAT scores and structural changes of the lungs in COPD patients.

\section{Materials and methods Subjects}

One hundred and twelve participants including 63 COPD patients and 49 normal control subjects (inclusion and exclusion criteria shown in Tables 1 and 2, respectively) were recruited. The COPD patients came from the Pulmonary Department, First Affiliated Hospital of Anhui Medical University, Hefei, Anhui, People's Republic of China, and the normal control subjects were all volunteers. Demographic information such as height, weight, age, and smoking history were recorded at recruitment. The study was approved by the ethics committee of the First Affiliated Hospital of Anhui Medical University. All participants had given written informed consent to participate in the study and were informed of the possible risks of the study. The study was performed from July 1, 2013 to February 28, 2014.

\section{Assessment of health status and dyspnea}

The health status was evaluated by CAT, which can also provide an assessment of symptoms burden. The degree of dyspnea was evaluated with the modified Medical Research Council (mMRC) questionnaire. All subjects completed CAT and mMRC questionnaires independently on the day of taking HRCT scans. The effect of COPD on health status was measured by the CAT scores, and the COPD patients

Table I Inclusion and exclusion criteria for COPD patients

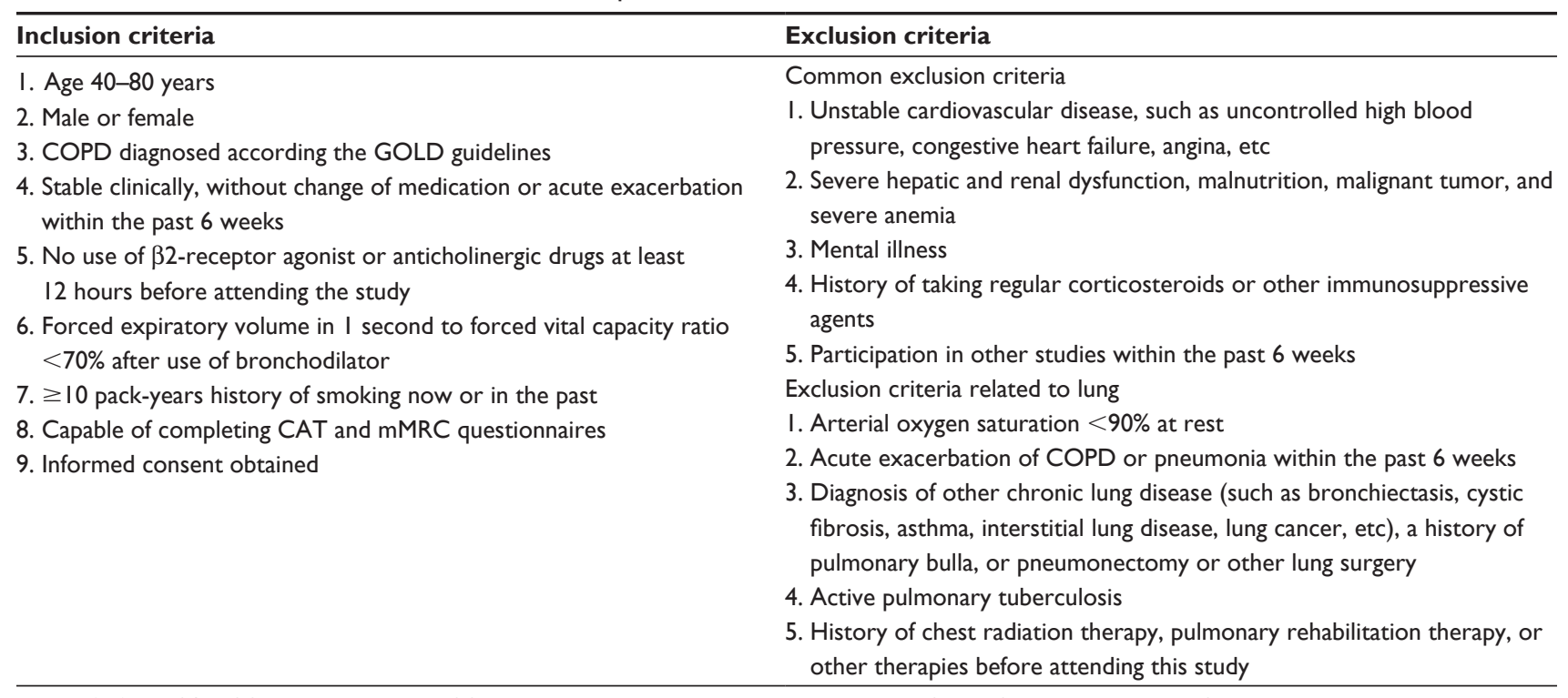

Abbreviations: CAT, COPD assessment test; COPD, chronic obstructive pulmonary disease; mMRC, modified Medical Research Council. 
Table 2 Inclusion and exclusion criteria for normal control subjects

\begin{tabular}{ll}
\hline Inclusion criteria & Exclusion criteria \\
\hline I. Age $40-80$ years & I. History of pulmonary disease or other system diseases (acute \\
2. Male or female & or chronic) \\
3. No illness, symptoms, or other discomfort & 2. Mental illness \\
4. Capable of completing CAT and mMRC questionnaires & 3. History of taking corticosteroids or immunosuppressive agents \\
5. Informed consent obtained & 4. Have ever participated or participating in any health care \\
& activities \\
\hline
\end{tabular}

were classified into group I (mild), II (moderate), III (severe), and IV (very severe) according to their CAT scores. ${ }^{3}$

\section{HRCT scans and analysis}

We measured emphysema and airway wall thickness using HRCT scans, which was performed using a 64-slice spiral CT scanner (Discovery HD750; GE, USA) at full inspiration without receiving contrast medium. Images were obtained at $120 \mathrm{kVp}$ and $30-60 \mathrm{mAs}$. The exposure time was 1 second and the matrix size was $512 \times 512$ pixels. Images were contiguously reconstructed using a standard algorithm with a slice thickness of $0.625 \mathrm{~mm}$ and a slice interval of $5 \mathrm{~mm}$. In transverse reconstructed images with a window width of 1,500 $\mathrm{HU}$ and window level of $-600 \mathrm{HU}$ on the workstation monitor. Reconstructed data were transferred to advantage workstation, and measurements of CT metrics were obtained with commercial software (Pulmonary Workstation; Vida Diagnostics, Coralville, IA, USA). All CT scans were reviewed by two experienced chest radiologists. Airway measurements were collected in the apical bronchus of the right upper lobe. \%WA was calculated as (total airway area - oairway lumen)/total airway area $\times 100$, and WT/D was calculated as airway wall thickness/outer diameter. ${ }^{18}$ Emphysema was defined as low attenuation areas using the HU threshold of $-950 ;{ }^{15,16}$ we calculated the percentage of pixels below this threshold (\%LAA-950) from the total number within lung parenchyma.

\section{Pulmonary function test}

Post-bronchodilator $\mathrm{FEV}_{1}$ was measured at 15 minutes after inhaling salbutamol (Ventolin; GlaxoSmithKline plc, London, UK) $400 \mu \mathrm{g}$, and the pulmonary function test was performed using a dry spirometer device (Erich Jaeger $\mathrm{GmbH}$, Hoechberg, Germany). Subjects with $\mathrm{FEV}_{1} / \mathrm{FVC}<0.70$ were defined as having COPD, and $\mathrm{FEV}_{1}$ higher than 50\% predicted were classified as GOLD I-II group; subjects with $\mathrm{FEV}_{1}$ lower than $50 \%$ predicted were classified as GOLD III-IV group according to the GOLD staging system. ${ }^{10}$

\section{Statistical analysis}

Demographic and clinical characteristics of all subjects were summarized descriptively. Data were expressed as mean \pm standard deviation. Data of CT measurements were all expressed as percentages. SPSS Statistics version 17.0 was used for statistical analysis. Group comparisons were tested using one-way analysis of variance. Differences between sex ratios were compared with the chi-square test. Pearson's correlation test was used to verify the relationship between numerical variables with normal distributions. The Spearman's rank correlation test was applied to nonparametric variables. Multiple linear stepwise regression analysis was used to explore the relationship between CAT scores and quantitative $\mathrm{CT}$ measurements. Values of $P<0.05$ were considered to be significant.

\section{Results \\ Demographic characteristics of all subjects}

One hundred and twelve participants were recruited, and the average age was $66.97 \pm 10.46$ years. The subjects were classified into three groups: GOLD I-II group ( $\mathrm{n}=30$ ), GOLD III-IV group $(n=33)$, and control group $(n=49)$. Table 3 shows the clinical characteristics of each group. The three groups were statistically similar with respect to age, sex, and body mass index (BMI) $(P>0.05)$, but the smoking index and mMRC grades were significantly higher in the COPD groups compared to the control subjects $(P<0.01)$, the pulmonary function parameters $\left(\mathrm{FEV}_{1} \%\right.$ and $\left.\mathrm{FEV}_{1} / \mathrm{FVC}\right)$ were significantly lower in the COPD groups $(P<0.01)$. Moreover, mMRC grade was significantly higher in the GOLD III-IV group compared to the GOLD I-II group $(P<0.01)$, and the pulmonary function parameters were significantly lower in the GOLD III-IV group compared to the GOLD I-II group $(P<0.01)$.

\section{Relationships between CAT scores and CT measurements in COPD patients}

The CAT scores and CT measurements of emphysema and airway wall thickness in COPD patients were all 
Table 3 Characteristics of all subjects

\begin{tabular}{lllll}
\hline Characteristic & Control group $\mathbf{n}=\mathbf{4 9}$ & GOLD I-II group $\mathbf{n = 3 0}$ & GOLD III-IV group $\mathbf{n}=\mathbf{3 3}$ & $\mathbf{P}$ \\
\hline Age, years & $65.1 \pm 10.3$ & $68.6 \pm 10 . I^{*}$ & $68.8 \pm I I .2^{*,+}$ & $11 / 22$ \\
Sex, female/male & $21 / 28$ & $13 / 17$ & $22.9 \pm 3.4^{*,+}$ & $0.20^{\mathrm{a}}$ \\
BMI $\left(\mathrm{kg} / \mathrm{m}^{2}\right)$ & $23.6 \pm 2.9$ & $23.2 \pm 3.2^{*}$ & $22.8 \pm 28.2^{* *,+}$ & $0.35^{\mathrm{b}}$ \\
Smoking index & $6.7 \pm 10.9$ & $17.6 \pm 18.4^{* * *}$ & $2.6 \pm 0.7^{* *,+}$ & $0.52^{\mathrm{a}}$ \\
mMRC grade & $0.1 \pm 0.2$ & $1.4 \pm 0.9^{* *}$ & $35.4 \pm 6.5^{* *,+}$ & $0.000^{\mathrm{a}}$ \\
FEV $\%$ & $100.3 \pm 13.7$ & $63.1 \pm 7.2^{* *}$ & $45.3 \pm 7.4^{* *,+}$ & $0.000^{\mathrm{a}}$ \\
FEV/FVC (\%) & $82.4 \pm 4.8$ & $63.9 \pm 4.0^{* *}$ & $0.000^{\mathrm{a}}$ \\
\hline
\end{tabular}

Notes: Data are presented as means \pm standard deviation. $P$-values were calculated by one-way analysis of variance, except where indicated otherwise. $* P>0.05$, $* * P<0.01$, $* * * P<0.05$ versus control group; ${ }^{+} P>0.05,{ }^{++} P<0.0$ I versus GOLD I-II group. ${ }^{a}$ One-way analysis of variance. ${ }^{b}$ Analysis of chi-square test.

Abbreviations: BMI, body mass index; mMRC, modified Medical Research Council.

significantly higher than in control subjects $(P<0.05)$, as shown in Table 4 . There was a tendency of higher CAT scores and $\mathrm{CT}$ measurements with increasing disease severity measured by GOLD staging system. CAT scores were taken as a dependent variable, while quantitative CT measurements, age, sex, and BMI were taken as independent variables in multiple linear stepwise regression analysis. Age, sex, BMI, and WT/D were not retained, but \%LAA950 and $\%$ WA were independent predictors of CAT score, giving the equation:

CAT score $=-46.38+0.778(\% \mathrm{WA})+0.203(\% \mathrm{LAA}-950)$

$\left(r^{2}=0.38, P=0.000\right)$. With the Pearson's correlation analysis, positive correlations were found between the CAT scores and \%LAA-950 ( $r=0.46, P=0.000$, Figure 1$), \% \mathrm{WA}$ $(r=0.48, P=0.000$, Figure 2$)$, and WT/D $(r=0.37, P=0.003$, Figure 3).

After classifying the COPD patients into four groups according to their CAT scores, 12 patients were group I (mild), 17 patients were group II (moderate), 25 patients were group III (severe), and nine patients were group IV (very severe). Table 5 shows the $\mathrm{CT}$ measurements in different CAT groups, and significant differences were found in \%LAA-950 and \%WA between group I and other groups $(P<0.05)$, but no difference was found in WT/D among CAT groups $(P>0.05)$.

\section{Relationships between pulmonary function and CT measurements, CAT scores in COPD patients}

The mean value of pulmonary function parameters $\left(\mathrm{FEV}_{1} \%\right.$ and $\mathrm{FEV}_{1} / \mathrm{FVC}$ ) in each group are shown in Table 3. The $\mathrm{FEV}_{1} \%$ predicted was inversely correlated with \%LAA-950 $(r=-0.65, P=0.000), \% \mathrm{WA}(r=-0.54, P=0.000)$, and WT/D $(r=-0.44, P=0.000)$. Similar relationships were also found between $\mathrm{FEV}_{1} / \mathrm{FVC}$ and \%LAA-950, \%WA, and WT/D ( $r=-0.60,-0.45$, and -0.37 , respectively, all $P=0.000$ ). CAT scores were inversely correlated with $\mathrm{FEV}_{1} \%(r=-0.63$, $P=0.000)$ and $\mathrm{FEV}_{1} / \mathrm{FVC}(r=-0.47, P=0.000)$.

\section{Relationships between mMRC grades and CT measurements, CAT scores in COPD patients}

With the Spearman's correlation analysis, the mMRC grades were positively correlated with $\%$ LAA $-950\left(r_{\mathrm{s}}=0.58\right.$, $P=0.000), \% \mathrm{WA}\left(r_{\mathrm{s}}=0.40, P=0.001\right)$, and WT/D $\left(r_{\mathrm{s}}=0.33\right.$, $P=0.008)$, as well as the CAT scores $\left(r_{\mathrm{s}}=0.66, P=0.000\right)$.

Table 4 CAT score and CT measurements in the total subjects

\begin{tabular}{lllll}
\hline & Control group & GOLD I-II group & GOLD III-IV group & P \\
\hline CAT score & $0.1 \pm 0.3$ & $15.1 \pm 8.8^{* *}$ & $24.9 \pm 7.0 * *,+$ & 0.000 \\
\%WA (\%) & $75.3 \pm 3.6$ & $77.9 \pm 5.6^{*}$ & $81.9 \pm 3.3 * *,+$ & 0.000 \\
WT/D (\%) & $25.8 \pm 2.5$ & $27.1 \pm 2.9 * *$ & $29.0 \pm 2.3^{* *,+}$ & 0.000 \\
\%LAA (\%) & $2.2 \pm 1.3$ & $7.0 \pm 2.8^{*}$ & $34.4 \pm 16.2^{* *,+}$ & 0.000 \\
\hline
\end{tabular}

Notes: Data are presented as means \pm standard deviation. $P$-values were calculated by analysis of variance. $* P<0.05, * * P<0.01$ versus control group; ${ }^{+} P<0.05,+P<0.0$ I versus GOLD I-II group.

Abbreviations: \%LAA, percentage of low attenuation areas; \%WA, wall area percentage; CAT, COPD assessment test; COPD, chronic obstructive pulmonary disease; CT, computed tomography; WT/D, ratio of airway wall thickness to total diameter. 


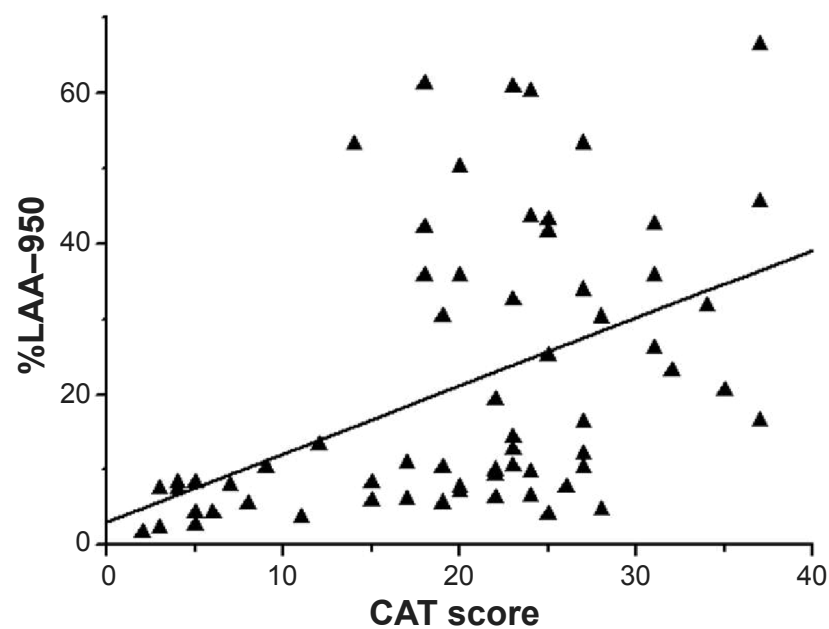

Figure I Positive correlation between the CAT score and \%LAA-950. Notes: $r=0.46, P=0.000$.

Abbreviations: \%LAA-950, percentage of pixels below -950 HU; CAT, chronic obstructive pulmonary disease assessment test.

\section{Discussion}

In the present study, we have demonstrated that the CAT scores and quantitative CT measurements of emphysema and airway wall thickness were significantly higher in COPD patients, which were consistent with the increasing disease severity measured by GOLD staging system. The CAT scores were significantly and positively correlated with the quantitative CT measurements in COPD patients. With a multiple linear stepwise regression analysis, both \%LAA-950 and \%WA were independent predictors of CAT scores. Meanwhile, both CAT scores and CT measurements were positively correlated to mMRC grades and inversely correlated to $\mathrm{FEV}_{1} \%$. After

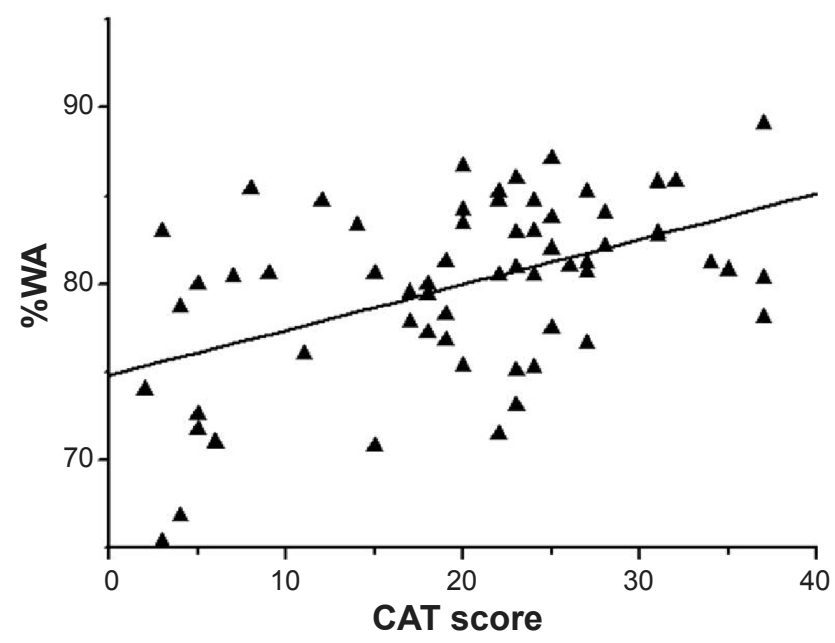

Figure 2 Positive correlation between the CAT score and \%WA. Notes: $r=0.48, P=0.000$.

Abbreviations: \%WA, wall area percentage; CAT, chronic obstructive pulmonary disease assessment test.

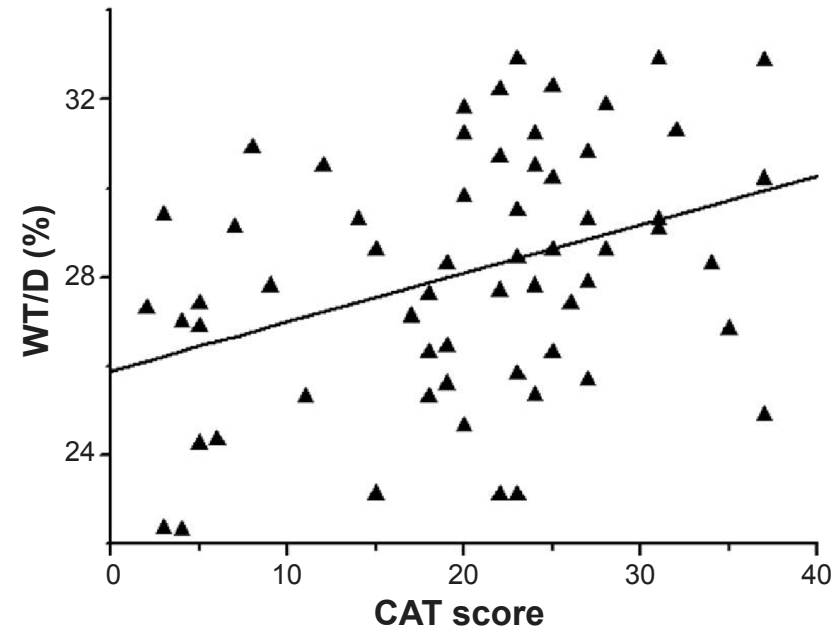

Figure 3 Positive correlation between the CAT score and WT/D. Notes: $r=0.37, P=0.003$.

Abbreviations: CAT, chronic obstructive pulmonary disease assessment test; WT/D, ratio of airway wall thickness to total diameter.

classifying the COPD patients according to the CAT scores, significant differences were found in \%WA and \%LAA-950 between group I and other groups.

COPD is a chronic inflammatory disease, and the chronic inflammatory responses can induce parenchymal tissue destruction (resulting in emphysema) and disrupt normal repair and defense mechanisms (resulting in small airway fibrosis). ${ }^{21}$ These pathological changes can lead to air trapping and progressive airflow limitation, which may directly cause dyspnea and other characteristic symptoms, as well as impaired health status. ${ }^{10}$ The CAT is a good measurement of health status and symptoms burden in COPD patients. ${ }^{1}$ Considering the CAT is a questionnaire completed by the patients, some problems such as subjectivity and being unilateral are inevitable during the examinations. While previous studies have shown the validated correlations between CAT scores and some other important parameters of COPD - such as dyspnea grades, pulmonary function parameters, and systemic inflammatory markers, ${ }^{7-9}$ which provided functional, physiologic, and serologic evidences for clinical practice of CAT - to our knowledge, correlation between CAT scores and the imaging parameters in COPD patients has not yet been carried out in the People's Republic of China.

Chest $\mathrm{CT}$ is the most prevalent examination to characterize the structural changes and offer various imaging parameters of lungs in clinic. The chest HRCT can not only give a more accurate quantification of airway dimensions and emphysema, but also allows for the phenotypic stratification of patients who have COPD into parenchymal- and 
Table 5 CT measurements in different CAT groups

\begin{tabular}{|c|c|c|c|c|c|c|}
\hline \multirow[t]{2}{*}{ CAT group } & \multicolumn{2}{|l|}{ \%WA (\%) } & \multicolumn{2}{|l|}{ WT/D (\%) } & \multicolumn{2}{|l|}{ \%LAA (\%) } \\
\hline & Mean \pm SD & $P$ & Mean \pm SD & $P$ & Mean \pm SD & $P$ \\
\hline \multirow[t]{2}{*}{ I $(0-10)$} & $75.8 \pm 6.4$ & & $26.6 \pm 2.7$ & & $5.8 \pm 2.8$ & \\
\hline & & 0.026 & & NS & & 0.009 \\
\hline \multirow[t]{2}{*}{ II (II-20) } & $79.7 \pm 4.0$ & & $27.5 \pm 2.4$ & & $22.8 \pm 19.7$ & \\
\hline & & NS & & NS & & NS \\
\hline \multirow[t]{2}{*}{ III $(2 \mathrm{I}-30)$} & $81.2 \pm 4.2$ & & $28.7 \pm 2.7$ & & $23.1 \pm 18.1$ & \\
\hline & & NS & & NS & & NS \\
\hline IV (3I-40) & $83.0 \pm 3.4$ & & $29.5 \pm 2.7$ & & $24.3 \pm 15.6$ & \\
\hline
\end{tabular}

Notes: Data are presented as means \pm SD. P-values were calculated by one-way analysis of variance.

Abbreviations: \%LAA, percentage of low attenuation areas; \%WA, wall area percentage; CAT, COPD assessment test; COPD, chronic obstructive pulmonary disease; $\mathrm{CT}$, computed tomography; NS, nonsignificant; SD, standard deviation; WT/D, ratio of airway wall thickness to total diameter.

airway-predominant categories. ${ }^{11-14,18}$ In COPD, the small airways ( $2 \mathrm{~mm}$ or less in diameter) are the site of major airflow resistance. ${ }^{10}$ Former studies have established that $\% \mathrm{WA}$ of the segment bronchus of the right upper lobe is a surrogate for the dimensions of those small airways measured by histological means, ${ }^{22}$ and that is also why the apical bronchus of the right upper lobe was chosen as our target measuring site in the present study. Madani et al reported that the best correlation between CT measurements and macroscopic and microscopic measures of emphysema can be obtained using a density mask technique with a threshold level of -960 or $-970 \mathrm{HU} .{ }^{23}$ In our study we chose the threshold of $-950 \mathrm{HU}$ in the inspiratory phase since we concerned that a threshold of -960 or -970 HU might exclude milder degrees of emphysema.

Previous studies have shown the relationships between quantitative $\mathrm{CT}$ measurements and respiratory symptoms in COPD patients. ${ }^{24-26}$ Grydeland et al found that both emphysema and airway wall thickness were independently and significantly related to the symptoms burden in COPD patients. ${ }^{24}$ A report from the COPDGene trial found that the extent of airway wall thickness was more strongly related to the symptoms burden than was emphysema. ${ }^{26} \mathrm{CAT}$ is also a good instrument to assess the symptoms burden. In the present study, we demonstrated that the CAT scores in COPD patients increased with the increasing level of emphysema and airway wall thickness measured by HRCT. Positive correlations were found between CAT scores and \%LAA-950, $\% \mathrm{WA}$, and WT/D, which might indicate that the change of symptoms burden correspond with the changes of quantitative CT measurements in COPD patients. Regression analysis showed that the independent predictors of CAT scores were \%LAA-950 and \%WA, but not WT/D; the specific reason for this difference is unknown, and further studies are needed to gain much more insight into answering this question. Interestingly, after classifying the COPD patients into four groups according to the CAT scores, significant differences were found only in \%WA and \%LAA-950 between group I (mild) and other groups. According to the GOLD guidelines, a CAT score $<10$ indicates a low level of symptoms, and a CAT score $\geq 10$ indicates a high level symptoms. ${ }^{10}$ A potential explanation for the differences in $\% \mathrm{WA}$ and \%LAA-950 between group I and other groups is that patients with a high level of symptoms may experience more serious destruction of lung parenchymal tissue and airway remodeling than the ones with less severe symptoms. While the WT/D is the ratio of airway wall thickness to total diameter, the differences might have been counterbalanced and caused the non-significant difference in WT/D between COPD patients with different levels of symptoms. However, more studies with large sample sizes are needed to gain more insight into these questions.

Dyspnea is usually the most prominent and distressing symptom of COPD patients. ${ }^{10}$ The correlations between dyspnea and imaging parameters in COPD patients were presented in several studies, ${ }^{20,24}$ as well as the association of dyspnea and CAT scores. ${ }^{9,27}$ Our study showed that both CT measurements and CAT scores were positively correlated with mMRC grades. Several explanations for these independent associations of the CT indices with dyspnea were discussed in previous studies. ${ }^{28,29}$ A number of studies have testified to the relationships between CT measurements and pulmonary function in COPD patients. ${ }^{18,19,30}$ Here we found that CT measurements and CAT scores were all negatively correlated with pulmonary function parameters; furthermore, the CT measurements and CAT scores all correspond well with the increasing disease severity measured by GOLD staging system, indicating that greater air trapping or thicker airway walls correlates with lower lung function, as well as more symptoms and worse health status. We speculate that 
both CAT and quantitative CT measurements might be used to partially reflect the extent of airflow limitation in COPD patients.

There are some limitations in this study. Firstly, only the apical bronchus of the right upper lobe was measured in this study, although previous studies have demonstrated that the apical bronchus of the right upper lobe can be a surrogate for the dimensions of the small airways. ${ }^{22}$ In the future, we need to collect more data for each lung lobe of COPD patients to obtain more accurate relationships between CAT scores and quantitative CT measurements. Secondly, the sample size in our study was relatively small, which may partially account for the weak correlations between some measurements in our results. Further prospective studies involving multicenter and large sample size of COPD patients are needed.

\section{Conclusion}

In conclusion, this study shows that CAT scores in COPD patients in the People's Republic of China correlate well with the quantitative CT measurements. Not only can it give assessments of health status or symptoms burden, but it can also be associated with the extent of emphysema and airway thickening in COPD patients. For the first time in the People's Republic of China, we provide an imaging evidence that the structural changes in lungs in COPD correlate with the health status measured by CAT. We suggest that a wide use of CAT may be performed in COPD patients in the People's Republic of China.

\section{Acknowledgments}

The authors would like to sincerely thank Rui Xu, Rong-huai $\mathrm{Li}$, and Xian-wei Hu for assistance with recruitment and clinical assessment of participants.

\section{Disclosure}

This work was supported by the National Science Foundation of China [No: 81270081]. The authors report no other conflicts of interest in this work.

\section{References}

1. Jones PW, Harding G, Berry P, Wiklund I, Chen WH, Kline Leidy N. Development and first validation of the COPD Assessment Test. Eur Respir J. 2009;34:648-654.

2. Jones P, Harding G, Wiklund I, Berry P, Leidy N. Improving the process and outcome of care in COPD: development of a standardised assessment tool. Prim Care Respir J. 2009;18(3):208-215.

3. COPD assessment test-healthcare professional user-guide [webpage on the Internet]. CAT Development Steering Group; 2009. Available from: http://www.catestonline.org/images/UserGuides. Accessed June 15, 2013.

4. Raghavan N, Lam YM, Webb KA, et al. Components of the COPD Assessment Test (CAT) associated with a diagnosis of COPD in a random population sample. COPD. 2012;9(2):175-183.
5. Dodd JW, Hogg L, Nolan J, et al. The COPD Assessment Test (CAT): response to pulmonary rehabilitation. A multicentre, prospective study. Thorax. 2011;66(5):425-429.

6. Jones PW, Harding G, Wiklund I, et al. Tests of the responsiveness of the COPD assessment test following acute exacerbation and pulmonary rehabilitation. Chest. 2012;142(1):134-140.

7. Tu YH, Zhang Y, Fei GH. Utility of the CAT in the therapy assessment of COPD exacerbations in China. BMC Pulm Med. 2014;14:42.

8. Mackay AJ, Donaldson GC, Patel AR, Jones PW, Hurst JR, Wedzicha JA. Usefulness of the Chronic Obstructive Pulmonary Disease Assessment Test to evaluate severity of COPD exacerbations. Am J Respir Crit Care Med. 2012;185 (11):1218-1224.

9. Okutan O, Tas D, Demirer E, Kartaloglu Z. Evaluation of quality of life with the chronic obstructive pulmonary disease assessment test in chronic obstructive pulmonary disease and the effect of dyspnea on diseasespecific quality of life in these patients. Yonsei Med J. 2013;54(5): 1214-1219.

10. Vestbo J, Hurd SS, Agusti AG, et al. Global strategy for the diagnosis, management and prevention of chronic obstructive pulmonary disease: GOLD executive summary. Am J Respir Crit Care Med. 2013; 187(4):347-365.

11. Lee YK, Oh YM, Lee JH, et al. Quantitative assessment of emphysema, air trapping, and airway thickening on computed tomography. Lung. 2008;186(3):157-165.

12. Van Tho N, Wada H, Ogawa E, Nakano Y. Recent findings in chronic obstructive pulmonary disease by using quantitative computed tomography. Respir Investig. 2012;50(3):78-87.

13. Matsuoka S, Yamashiro T, Washko GR, Kurihara Y, Nakajima Y, Hatabu H. Quantitative CT assessment of chronic obstructive pulmonary disease. Radiographics. 2010;30:55-66.

14. Orlandi I, Moroni C, Camiciottoli G, et al. Chronic obstructive pulmonary disease: thin-section CT measurement of airway wall thickness and lung attenuation. Radiology. 2005;234:604-610.

15. Parr DG, Stoel BC, Stolk J, Nightingale PG, Stockley RA. Influence of calibration on densitometric studies of emphysema progression using computed tomography. Am J Respir Crit Care Med. 2004;170(8): $883-890$.

16. Gevenois PA, de Maertelaer V, De Vuyst P, Zanen J, Yernault JC. Comparison of computed density and macroscopic morphometry in pulmonary emphysema. Am J Respir Crit Care Med. 1995;152: 653-657.

17. Coxson HO, Quiney B, Sin DD, et al. Airway wall thickness assessed using computed tomography and optical coherence tomography. Am J Respir Crit Care Med. 2008;177:1201-1206.

18. Kosciuch J, Krenke R, Gorska K, Zukowska M, MaskeyWarzechowska M, Chazan R. Relationship between airway wall thickness assessed by high-resolution computed tomography and lung function in patients with asthma and chronic obstructive pulmonary disease. J Physiol Pharmacol. 2009;60 Suppl 5:71-76.

19. Mets OM, Murphy K, Zanen P, et al. The relationship between lung function impairment and quantitative computed tomography in chronic obstructive pulmonary disease. Eur Radiol. 2012;22(1):120-128.

20. Camiciottoli G, Bartolucci M, Maluccio NM, et al. Spirometrically gated high-resolution CT findings in COPD: lung attenuation vs lung function and dyspnea severity. Chest. 2006;129:558-564.

21. Hogg JC. Pathophysiology of airflow limitation in chronic obstructive pulmonary disease. Lancet. 2004;364:709-721.

22. Nakano Y, Wong JC, de Jong PA, et al. The prediction of small airway dimensions using computed tomography. Am J Respir Crit Care Med. 2005;171:142-146.

23. Madani A, Zanen J, de Maertelaer V, Gevenois PA. Pulmonary emphysema: objective quantification at multi-detector row CT - comparison with macroscopic and microscopic morphometry. Radiology. 2006;238:1036-1043.

24. Grydeland TB, Dirksen A, Coxson HO, et al. Quantitative computed tomography measures of emphysema and airway wall thickness are related to respiratory symptoms. Am J Respir Crit Care Med.2010;181(4): 353-359. 
25. Han MK, Bartholmai B, Liu LX, et al. Clinical significance of radiologic characterizations in COPD. COPD. 2009;6(6):459-467.

26. Martinez $\mathrm{CH}$, Chen $\mathrm{YH}$, Westgate PM, et al. Relationship between quantitative $\mathrm{CT}$ metrics and health status and BODE in chronic obstructive pulmonary disease. Thorax. 2012;67(5):399-406.

27. Zhou QT, Mei JJ, He B, et al. Chronic obstructive pulmonary disease assessment test score correlated with dyspnea score in a large sample of Chinese patients. Chin Med J (Eng). 2013;126(1):11-15.
28. O'Donnell DE, Laveneziana P. Dyspnea and activity limitation in COPD: mechanical factors. COPD. 2007;4:225-236.

29. Wouters EF. Local and systemic inflammation in chronic obstructive pulmonary disease. Proc Am Thorac Soc. 2005;2:26-33.

30. Schroeder JD, McKenzie AS, Zach JA, et al. Relationships between airflow obstruction and quantitative CT measurements of emphysema, air trapping, and airways in subjects with and without chronic obstructive pulmonary disease. ARJ Am J Roentgenol. 2013;201(3):W460-W470.

\section{Publish your work in this journal}

The International Journal of COPD is an international, peer-reviewed journal of therapeutics and pharmacology focusing on concise rapid reporting of clinical studies and reviews in COPD. Special focus is given to the pathophysiological processes underlying the disease, intervention programs, patient focused education, and self management protocols.

\section{Dovepress}

This journal is indexed on PubMed Central, MedLine and CAS. The manuscript management system is completely online and includes a very quick and fair peer-review system, which is all easy to use. Visit $\mathrm{http} / / / \mathrm{www}$.dovepress.com/testimonials.php to read real quotes from published authors.

\footnotetext{
Submit your manuscript here: http://www.dovepress.com/international-journal-of-chronic-obstructive-pulmonary-disease-journal
} 